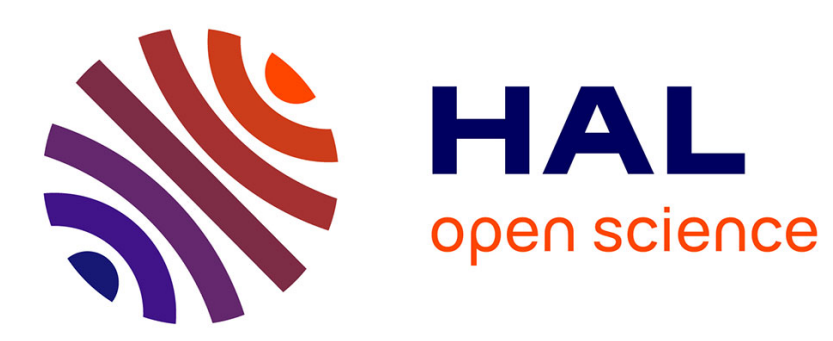

\title{
Identification of space deformation using linear and superficial quadratic variations
}

\author{
Xavier Guyon, Olivier Perrin
}

\section{To cite this version:}

Xavier Guyon, Olivier Perrin. Identification of space deformation using linear and superficial quadratic variations. Statistics and Probability Letters, 2000, 47 (3), pp.307-316. 10.1016/S01677152(99)00171-6 . hal-00273111

\section{HAL Id: hal-00273111 \\ https://hal.science/hal-00273111}

Submitted on 14 Apr 2008

HAL is a multi-disciplinary open access archive for the deposit and dissemination of scientific research documents, whether they are published or not. The documents may come from teaching and research institutions in France or abroad, or from public or private research centers.
L'archive ouverte pluridisciplinaire HAL, est destinée au dépôt et à la diffusion de documents scientifiques de niveau recherche, publiés ou non, émanant des établissements d'enseignement et de recherche français ou étrangers, des laboratoires publics ou privés. 


\title{
Identification of space deformation using linear and superficial quadratic variations
}

\author{
Xavier Guyon*and Olivier Perrin ${ }^{\dagger}$ \\ SAMOS, Université Paris I, France
}

August 24, 1999

\begin{abstract}
We use linear and superficial quadratic variations to identify a bijective space deformation that makes a non-stationary Gaussian random field stationary.
\end{abstract}

keywords: identification of a model; mean square convergence; quadratic variations; space deformation; stationary reducibility

\section{Introduction}

In spatial statistics we are often concerned with non-stationary phenomena. For most applications dealing with a non-stationary Gaussian random field, the first step in classical approaches consists of removing expectation, dividing the residual by the standard deviation and modelling the residual as a stationary process. That is to say, the random field $Y=\left\{Y(x, y):(x, y) \in G \subseteq \mathbb{R}^{2}\right\}$ (where $G$ stands for $G$ eographical space) under study is of the form

$$
Y(x, y)=\mu(x, y)+\sigma(x, y) Z(x, y)
$$

where $\mu(x, y)=E Y(x, y), \sigma(x, y)^{2}=E(Y(x, y)-\mu(x, y))^{2}$ and $Z(x, y)$ is a reduced (centred and standardised) stationary Gaussian random field. Then the non-stationarity of the random field $Y$ is simply understood as non-stationarity of both the first order moment $\mu(x, y)$ and the standard deviation $\sigma(x, y)$. Nevertheless, $Z$ can also be non-stationary. In this case, Sampson and Guttorp [11] propose a transformation of the index space $G$ with a bijective space deformation. Formally, it consists of modelling $Z(x, y)$ as

$$
Z(x, y)=\delta(\Phi(x, y))
$$

${ }^{*}$ SAMOS, Université Paris I, 90, rue de Tolbiac, 75634 Paris Cedex 13, France, E-mail: guyon@univ-paris1.fr

${ }^{\dagger}$ Mathematical Statistics, Chalmers University of Technology and Göteborg University, S-412 96 Göteborg, Sweden, E-mail: olivier@math.chalmers.se 
where $\delta$ is stationary and $\Phi=\left(\Phi_{1}, \Phi_{2}\right): G \longrightarrow D$ (where $D$ stands for $D$ eformed space) is a bijective deformation. Equivalently, the correlation function $r$ of $Z$ is

$$
r\left(x, y, x^{\prime}, y^{\prime}\right)=R\left(\Phi\left(x^{\prime}, y^{\prime}\right)-\Phi(x, y)\right)
$$

where $R$ is a stationary correlation function in $\mathbb{R}^{2}$.

In this paper, we consider a reduced Gaussian random field $Z$ indexed by $G=[0,1]^{2}$ satisfying (1)-(2) and we suppose that the stationary correlation $R$ is known. Our concern is the functional estimation of the space deformation $\Phi$ using a set of suitable linear and superficial quadratic variations of $Z$.

The quadratic variations are first introduced by Lévy [8] who shows that if $Z$ is the standard Wiener process on $[0,1]$, then almost surely its quadratic variation on $[0,1]$ converges to 1 . Baxter [2] and further Gladyshev [3] generalise this result to a large class of Gaussian processes. Guyon and León [5] introduce the $H$-variations for stationary Gaussian processes, a generalisation of these quadratic variations. They study the convergence in distribution of the $H$ variations, suitably normalised.

For Gaussian process $Z$ with stationary increments, Istas and Lang [6] define general quadratic variations, substituting a general discrete difference operator to the simple difference $Z(k / n)-Z((k-1) / n)$. They use these quadratic variations to estimate the Hölder index of a process.

For non-stationary Gaussian processes, with increments stationary or not, Perrin [9] gives a general result concerning the functional asymptotic normality of the process of the quadratic variations which corresponds to the linear interpolation of the points $\left(p / n, V_{n}(p / n)\right), p=1,2, \ldots, n$, with $V_{n}(p / n)$ the discrete quadratic variations at points $p / n$. This result is applied to the estimation of a time deformation for non-stationary models of the form $Z(x)=\delta(\Phi(x)), \quad x \in$ $[0,1]$.

The generalisation of quadratic variations for stationary Gaussian fields is studied in Guyon [4] and León and Ortega [7]. Another generalisation for nonstationary Gaussian processes and quadratic variations along curves is done in Adler and Pyke [1]. Guyon [4] shows that some stationary random fields can be identified in mean square sense using different families of variations. Using some of these families allows us, in this paper, to generalise the result of [9] to the estimation of a space deformation for non-stationary models of the form (1)-(2).

The paper is structured as follows. Section 2 sets up notations, assumptions, definitions and describes the superficial and the linear quadratic variations. In Section 3, we study the pointwise mean square $\left(L_{2}\right)$ convergence of these quadratic variations. In Section 4 , we propose an estimator of $\Phi$ which converges in $L_{2}$ to $\Phi$. This estimator is defined with the help of the superficial and the linear quadratic variations. Finally, Section 5 discusses two extensions of the present work for future research.

\section{Linear and Superficial quadratic variations}

Let $Z=\left\{Z(x, y),(x, y) \in[0,1]^{2}\right\}$ be a real-valued reduced Gaussian random field with correlation $r$ satisfying (2). 
For any differentiable function $f:(x, y) \in[0,1]^{2} \rightarrow \mathbb{R}$, we denote using $f^{\left(p_{1}, p_{2}\right)}$ the $p_{1}, p_{2}$-partial derivative of $f$ with respect to $x$ and $y$. Assume for the stationary correlation function $R$

(A1) $R(u, v)$ satisfies when $u \rightarrow 0$ and $v \rightarrow 0$ : $R(u, v)=1-\alpha|u|-\beta|v|+O(u v), \quad$ where $\alpha>0$ and $\beta>0$.

(A2) $R^{(2,0)}(u, v), R^{(1,1)}(u, v), R^{(0,2)}(u, v)$ exist and are uniformly bounded in $\{(u, v): u \neq 0\},\{(u, v): u v \neq 0\},\{(u, v): v \neq 0\}$.

For instance, the stationary exponential model $R(u, v)=\exp (-\alpha|u|-\beta|v|)$ satisfies (A1)-(A2).

We consider the following smoothness assumption for the deformation $\Phi$

(B1) $\Phi$ has uniformly bounded second derivatives in $[0,1]^{2}$.

We restrict to bijective deformations $\Phi=\left(\Phi_{1}, \Phi_{2}\right)$ such that $\Phi_{1}$ and $\Phi_{2}$ satisfy the following assumptions for all $(x, y) \in[0,1]^{2}$

$$
\Phi_{1}^{(1,0)}(x, y)>0, \Phi_{2}^{(0,1)}(x, y)>0, \Phi_{1}^{(0,1)}(x, y) \geq 0, \Phi_{2}^{(1,0)}(x, y) \geq 0 .
$$

Moreover, it is reasonable to assume that the Jacobian determinant of $\Phi$ is strictly positive in $[0,1]^{2}$. In particular, it follows from (B2) that for all $(x, y) \in$ $[0,1]^{2}$ we have

$$
\frac{\Phi_{1}^{(0,1)}(x, y)}{\Phi_{1}^{(1,0)}(x, y)}<\frac{\Phi_{2}^{(0,1)}(x, y)}{\Phi_{2}^{(1,0)}(x, y)}
$$

We strengthen this condition by

$$
\text { (B3) } a=\sup _{(x, y) \in[0,1]^{2}} \frac{\Phi_{1}^{(0,1)}(x, y)}{\Phi_{1}^{(1,0)}(x, y)}<\inf _{(x, y) \in[0,1]^{2}} \frac{\Phi_{2}^{(0,1)}(x, y)}{\Phi_{2}^{(1,0)}(x, y)}=b \text {. }
$$

It is easy to see ( $c f$. [10]) that if $(\Phi, R)$ is a solution to (2), then any other solution $(\tilde{\Phi}, \tilde{R})$ is of the form $\tilde{\Phi}(x)=B \Phi(x, y)+b$ and $\tilde{R}(u)=R\left(B^{-1} u\right)$, where $B$ is a regular square matrix and $b$ is a vector in $\mathbb{R}^{2}$. Thus, without loss of generality, we may impose that

$$
\Phi(0)=0 .
$$

Bijections $\Phi=\left(\Phi_{1}, \Phi_{2}\right)$ such that $\Phi_{1}(x, y)=F(x)$ and $\Phi_{2}(x, y)=G(y)$ for all $(x, y) \in[0,1]^{2}$, where $F$ and $G$ are two twice continuously differentiable strictly increasing functions in $[0,1]$, as are $F^{-1}$ and $G^{-1}$, satisfy (B1)-(B3) with $a=0$ and $b=\infty$. More generally, twice continuously differentiable bijections $\Phi=\left(\Phi_{1}, \Phi_{2}\right)$ in $[0,1]^{2}$, as are $\Phi^{-1}$, with strictly positive first partial derivatives and where $\Phi_{1}$ does not depend on $y$ (respectively $\Phi_{2}$ does not depend on $x$ ) satisfy (B1)-(B3) with $a=0$ (respectively $b=\infty$ ). Bijections of the form

$$
\left(\begin{array}{c}
x \\
y
\end{array}\right) \longmapsto\left(\begin{array}{c}
c_{1} x+c_{2} y+c_{3} x y \\
d_{1} x+d_{2} y+d_{3} x y
\end{array}\right)
$$


where $c_{1}>0, c_{2} \geq 0, c_{3} \geq 0, d_{1} \geq 0, d_{2}>0, d_{3} \geq 0$ and $\left(c_{2}+c_{3}\right)\left(d_{1}+d_{3}\right)<c_{1} d_{2}$, satisfy (B1)-(B3). These deformations transform lines onto lines and reduce to linear transformations when $c_{3}=d_{3}=0$. In this case $Z$ is already a stationary process with correlation function $\tilde{R}(u, v)=R((u, v) M)$ where $M=\left(\begin{array}{ll}c_{1} & d_{1} \\ c_{2} & d_{2}\end{array}\right)$.

Let $n$ and $m$ be two positive integers, $\Pi_{n, m}$ the product partition of $[0,1]^{2}$ with mesh $1 / n$ in $x$ and mesh $1 / m$ in $y$. We call $\lambda=\frac{m}{n}$ the geometry of $\Pi_{n, m}$. Note that $\lambda$ is a parameter under our control. We define the rectangular increment $Z(\Delta)=Z\left(x^{\prime}, y^{\prime}\right)-Z\left(x^{\prime}, y\right)-Z\left(x, y^{\prime}\right)+Z(x, y)$ for the rectangle $\Delta=\left[(x, y),\left(x^{\prime}, y^{\prime}\right)\left[\right.\right.$, with $0 \leq x<x^{\prime} \leq 1$ and $0 \leq y<y^{\prime} \leq 1$. Then we define for $k=0,1, \ldots, n-1$ and $y \in[0,1]$

$$
\Delta_{k, y}=\left[\left(k / n,\left\lfloor m\left(y \wedge \frac{m-1}{m}\right)\right\rfloor / m\right),\left((k+1) / n,\left(\left\lfloor m\left(y \wedge \frac{m-1}{m}\right)\right\rfloor+1\right) / m\right)[,\right.
$$

where $\lfloor u\rfloor$ denotes the greatest integer smaller than or equal to $u$, and $u \wedge v$ denotes the minimum of $u$ and $v$. We define $\Delta_{x, l}$ in a symmetric way for $l=0,1, \ldots, m-1$ and $x \in[0,1]$

$$
\Delta_{x, l}=\left[\left(\left\lfloor n\left(x \wedge \frac{n-1}{n}\right)\right\rfloor / n, l / m\right),\left(\left(\left\lfloor n\left(x \wedge \frac{n-1}{n}\right)\right\rfloor+1\right) / n,(l+1) / m\right)[.\right.
$$

We also consider the following one-dimensional $H$ orizontal and $V$ ertical increments for $k=0,1, \ldots, n-1$ and $l=0,1, \ldots, m-1$

$$
Z\left(\Delta_{k}^{H}\right)=Z\left(\frac{k+1}{n}, 0\right)-Z\left(\frac{k}{n}, 0\right) \text { and } Z\left(\Delta_{l}^{V}\right)=Z\left(0, \frac{l+1}{m}\right)-Z\left(0, \frac{l}{m}\right) .
$$

We define for all $(x, y) \in[0,1]^{2}$ two superficial quadratic variations, $H_{n, \lambda}(x, y)$ (in $x$ at fixed level $y$ ) and $V_{m, \lambda}(x, y)$ (in $y$ at fixed level $x$ ) as follows

$$
H_{n, \lambda}(x, y)=\sum_{k=0}^{\lfloor n x\rfloor-1}\left(Z\left(\Delta_{k, y}\right)\right)^{2} \text { and } V_{m, \lambda}(x, y)=\sum_{l=0}^{\lfloor m y\rfloor-1}\left(Z\left(\Delta_{x, l}\right)\right)^{2} \text {. }
$$

We also define two linear quadratic variations $h_{n}(x), x \in[0,1]$, and $v_{m}(y)$, $y \in[0,1]$, as follows

$$
h_{n}(x)=\sum_{k=0}^{\lfloor n x\rfloor-1}\left(Z\left(\Delta_{k}^{H}\right)\right)^{2} \text { and } v_{m}(y)=\sum_{l=0}^{\lfloor m y\rfloor-1}\left(Z\left(\Delta_{l}^{V}\right)\right)^{2} .
$$

When $\lfloor n x\rfloor=0$ (respectively $\lfloor m y\rfloor=0$ ) we set $H_{n, \lambda}(x, y)=0$ and $h_{n}(x)=0$ (respectively $V_{m, \lambda}(x, y)=0$ and $v_{m}(y)=0$ ).

\section{Asymptotic properties}

In this section, we are concerned with asymptotic properties of the quadratic variations defined in the previous section. Because of the symmetry in the definitions of $H_{n, \lambda}(x, y)$ and $V_{m, \lambda}(x, y)$ (respectively $h_{n}(x)$ and $v_{m}(y)$ ), we focus our attention on $H_{n, \lambda}(x, y)$ and $h_{n}(x)$. 


\subsection{Convergence of the means}

\subsubsection{Superficial quadratic variations}

Denote using $\mathbb{Q}^{+}$the set of the strictly positive rational numbers. Define for all $(x, y) \in[0,1]^{2}$ and any $\lambda \in \mathbb{Q}^{+}$

$$
\begin{aligned}
& H_{\lambda}(x, y)=4\left(\beta\left(\Phi_{2}(x, y)-\Phi_{2}(0, y)\right)+\frac{\alpha}{\lambda} \int_{0}^{x} \Phi_{1}^{(0,1)}(u, y) d u\right) \\
& V_{\lambda}(x, y)=4\left(\beta \lambda \int_{0}^{y} \Phi_{2}^{(1,0)}(x, v) d v+\alpha\left(\Phi_{1}(x, y)-\Phi_{1}(x, 0)\right)\right) .
\end{aligned}
$$

We first establish the following lemma

Lemma 3.1 Assume (B1)-(B3). Then for all $(x, y) \in[0,1]^{2}$ such that $(x+1 / n, y+1 / m) \in[0,1]^{2}$ and any $\left.\lambda=\frac{m}{n} \in\right] a, b\left[\cap \mathbb{Q}^{+}\right.$

$$
\Phi_{1}(x+1 / n, y) \geq \Phi_{1}(x, y+1 / m) \text { and } \Phi_{2}(x, y+1 / m) \geq \Phi_{2}(x+1 / n, y),
$$

as $n \rightarrow \infty$ and $m \rightarrow \infty$.

Proof. Under assumption (B1), a Taylor expansion of order one with Lagrange remainder gives for all $(x, y) \in[0,1]^{2}$ such that $(x+1 / n, y+1 / m) \in[0,1]^{2}$

$$
\Phi_{1}(x+1 / n, y)-\Phi_{1}(x, y+1 / m)=\frac{1}{n} \Phi_{1}^{(1,0)}(x, y)-\frac{1}{m} \Phi_{1}^{(0,1)}(x, y)+O\left(\frac{1}{m^{2}}\right),
$$

where $O($.$) is uniform in both x$ and $y$. Then from (B2) and (B3) we obtain

$$
\Phi_{1}(x+1 / n, y)-\Phi_{1}(x, y+1 / m) \geq \frac{1}{m} \Phi_{1}^{(1,0)}(x, y)(\lambda-a)+O\left(\frac{1}{m^{2}}\right) .
$$

Thus, for any $\lambda \in] a, b\left[\cap \mathbb{Q}^{+}, \Phi_{1}(x+1 / n, y)-\Phi_{1}(x, y+1 / m) \geq 0\right.$ as $m \rightarrow \infty$.

Under assumptions (B1)-(B3), we obtain for all $(x, y) \in[0,1]^{2}$ such that $(x+1 / n, y+1 / m) \in[0,1]^{2}$

$$
\Phi_{2}(x, y+1 / m)-\Phi_{2}(x+1 / n, y) \geq \frac{1}{n} \Phi_{2}^{(0,1)}(x, y)\left(\frac{1}{\lambda}-\frac{1}{b}\right)+O\left(\frac{1}{n^{2}}\right),
$$

where $O($.$) is uniform in both x$ and $y$. Thus, for any $\lambda \in] a, b\left[\cap \mathbb{Q}^{+}, \Phi_{2}(x, y+\right.$ $1 / m)-\Phi_{2}(x+1 / n, y) \geq 0$ as $n \rightarrow \infty$.

Theorem 3.1 Assume (A1) and (B1)-(B3). Then for all $(x, y) \in[0,1]^{2}$ and any $\lambda \in] a, b\left[\cap \mathbb{Q}^{+}\right.$

(i) $\lim _{n \rightarrow \infty} E\left(H_{n, \lambda}(x, y)\right)=H_{\lambda}(x, y)$ and $(i i) \lim _{m \rightarrow \infty} E\left(V_{m, \lambda}(x, y)\right)=V_{\lambda}(x, y)$. 


\section{Proof.}

(i) We set $y^{\prime}=y \wedge \frac{m-1}{m}$ for all $y \in[0,1]$ and

$$
\begin{array}{ll}
A=\left(k / n,\left\lfloor m y^{\prime}\right\rfloor / m\right), & B=\left((k+1) / n,\left\lfloor m y^{\prime}\right\rfloor / m\right), \\
C=\left(k / n,\left(\left\lfloor m y^{\prime}\right\rfloor+1\right) / m\right), & D=\left((k+1) / n,\left(\left\lfloor m y^{\prime}\right\rfloor+1\right) / m\right),
\end{array}
$$

for $k=0,1, \ldots, n-1$. We have for all $(x, y) \in[0,1]^{2}: E\left(H_{n, \lambda}(x, y)\right)=$ $\sum_{k=0}^{\lfloor n x\rfloor-1} E\left(Z\left(\Delta_{k, y}\right)\right)^{2}$ with

$$
\begin{gathered}
E\left(Z\left(\Delta_{k, y}\right)\right)^{2}=4+2\{R(\Phi(D)-\Phi(A))+R(\Phi(B)-\Phi(C))-R(\Phi(B)-\Phi(A)) \\
-R(\Phi(C)-\Phi(A))-R(\Phi(D)-\Phi(B))-R(\Phi(D)-\Phi(C))\} .
\end{gathered}
$$

From (A1), (B2) and Lemma (3.1) we get as $n \rightarrow \infty$ and $m \rightarrow \infty$

$$
\begin{aligned}
E\left(Z\left(\Delta_{k, y}\right)\right)^{2} & =2 \alpha\left(\Phi_{1}(D)-\Phi_{1}(A)-\Phi_{1}(B)+\Phi_{1}(C)\right) \\
& +2 \beta\left(\Phi_{2}(D)-\Phi_{2}(A)+\Phi_{2}(B)-\Phi_{2}(C)\right) \\
& +O\left(\left(\Phi_{1}(D)-\Phi_{1}(A)\right)\left(\Phi_{2}(D)-\Phi_{2}(A)\right)\right) .
\end{aligned}
$$

Under assumption (B1), a Taylor expansion of order one for both $\Phi_{1}$ and $\Phi_{2}$ gives

$$
\begin{aligned}
& E\left(Z\left(\Delta_{k, y}\right)\right)^{2} \\
& \quad=\frac{4}{n}\left(\beta \Phi_{2}^{(1,0)}\left(\frac{k}{n}, \frac{\left\lfloor m y^{\prime}\right\rfloor}{m}\right)+\frac{\alpha}{\lambda} \Phi_{1}^{(0,1)}\left(\frac{k}{n}, \frac{\left\lfloor m y^{\prime}\right\rfloor}{m}\right)\right)+O\left(\frac{1}{n m}\right),
\end{aligned}
$$

where $O($.$) is uniform in both k$ and $y$. In addition, a Taylor expansion of order 0 gives

$$
\begin{aligned}
& \Phi_{1}^{(0,1)}\left(\frac{k}{n}, \frac{\left\lfloor m y^{\prime}\right\rfloor}{m}\right)=\Phi_{1}^{(0,1)}\left(\frac{k}{n}, y\right)+O\left(\frac{1}{m}\right) \\
& \Phi_{2}^{(1,0)}\left(\frac{k}{n}, \frac{\left\lfloor m y^{\prime}\right\rfloor}{m}\right)=\Phi_{2}^{(1,0)}\left(\frac{k}{n}, y\right)+O\left(\frac{1}{m}\right) .
\end{aligned}
$$

Thus

$$
E\left(H_{n, \lambda}(x, y)\right)=\frac{4}{n} \sum_{k=0}^{\lfloor n x\rfloor-1}\left(\beta \Phi_{2}^{(1,0)}\left(\frac{k}{n}, y\right)+\frac{\alpha}{\lambda} \Phi_{1}^{(0,1)}\left(\frac{k}{n}, y\right)\right)+O\left(\frac{1}{n}\right) .
$$

Since $\Phi_{2}^{(1,0)}(., y)$ and $\Phi_{1}^{(0,1)}(., y)$ are Riemann integrable in $[0,1]$ for all $y \in[0,1]$, we get for all $(x, y) \in[0,1]^{2}$ and for any $\left.\lambda \in\right] a, b\left[\cap \mathbb{Q}^{+}\right.$

$$
\lim _{n \rightarrow \infty} E\left(H_{n, \lambda}(x, y)\right)=4\left(\beta\left(\Phi_{2}(x, y)-\Phi_{2}(0, y)\right)+\frac{\alpha}{\lambda} \int_{0}^{x} \Phi_{1}^{(0,1)}(u, y) d u\right) .
$$

(ii) We get for all $(x, y) \in[0,1]^{2}$

$$
E\left(V_{m, \lambda}(x, y)\right)=\frac{4}{m} \sum_{l=0}^{\lfloor m y\rfloor-1}\left(\lambda \beta \Phi_{2}^{(1,0)}\left(x, \frac{l}{m}\right)+\alpha \Phi_{1}^{(0,1)}\left(x, \frac{l}{m}\right)\right)+O\left(\frac{1}{m}\right),
$$


so that for any $\lambda \in] a, b\left[\cap \mathbb{Q}^{+}\right.$

$$
\lim _{m \rightarrow \infty} E\left(V_{m, \lambda}(x, y)\right)=4\left(\beta \lambda \int_{0}^{y} \Phi_{2}^{(1,0)}(x, v) d v+\alpha\left(\Phi_{1}(x, y)-\Phi_{1}(x, 0)\right)\right) .
$$

\subsubsection{Linear quadratic variations}

Define for all $(x, y) \in[0,1]^{2}$

$$
h(x)=2\left(\alpha \Phi_{1}(x, 0)+\beta \Phi_{2}(x, 0)\right) \text { and } v(y)=2\left(\alpha \Phi_{1}(0, y)+\beta \Phi_{2}(0, y)\right) .
$$

Theorem 3.2 Assume (A1) and (B1)-(B2). Then for all $(x, y) \in[0,1]^{2}$

(i) $\lim _{n \rightarrow \infty} E\left(h_{n}(x)\right)=h(x)$ and $(i i) \lim _{m \rightarrow \infty} E\left(v_{m}(y)\right)=v(y)$.

\section{Proof.}

(i) For all $x \in[0,1]: E\left(h_{n}(x)\right)=\sum_{k=0}^{\lfloor n x\rfloor-1} E\left(Z\left(\Delta_{k}^{H}\right)\right)^{2}$. From (A1) and (B1)(B2) we have as $n \rightarrow \infty$

$$
E\left(Z\left(\Delta_{k}^{H}\right)\right)^{2}=2 \alpha \Phi_{1}\left(\Delta_{k}^{H}\right)+2 \beta \Phi_{2}\left(\Delta_{k}^{H}\right)+O\left(\Phi_{1}\left(\Delta_{k}^{H}\right) \Phi_{2}\left(\Delta_{k}^{H}\right)\right) .
$$

A Taylor expansion of order one for both $\Phi_{1}$ and $\Phi_{2}$ gives

$$
E\left(Z\left(\Delta_{k}^{H}\right)\right)^{2}=\frac{2}{n}\left(\alpha \Phi_{1}^{(1,0)}\left(\frac{k}{n}, 0\right)+\beta \Phi_{2}^{(1,0)}\left(\frac{k}{n}, 0\right)\right)+O\left(\frac{1}{n^{2}}\right),
$$

where $O($.$) is uniform in k$. Therefore, we get for all $x \in[0,1]$

$$
E\left(h_{n}(x)\right)=\frac{2}{n} \sum_{k=0}^{\lfloor n x\rfloor-1}\left(\alpha \Phi_{1}^{(1,0)}\left(\frac{k}{n}, 0\right)+\beta \Phi_{2}^{(1,0)}\left(\frac{k}{n}, 0\right)\right)+O\left(\frac{1}{n}\right) .
$$

$\Phi_{1}^{(1,0)}(., 0)$ and $\Phi_{2}^{(1,0)}(., 0)$ being Riemann integrable in [0,1], it follows from (3) that the right-hand side on (8) converges to $2\left(\alpha \Phi_{1}(x, 0)+\beta \Phi_{2}(x, 0)\right)$ as $n \rightarrow \infty$.

A similar treatment holds for (ii).

\section{2 $L_{2}$ convergence of the variations}

\subsubsection{Superficial quadratic variations}

Theorem 3.3 Assume (A1)-(A2) and (B1)-(B3). Then for all $(x, y) \in$ $[0,1]^{2}$ and any $\left.\lambda \in\right] a, b\left[\cap \mathbb{Q}^{+}\right.$

(i) $\lim _{n \rightarrow \infty} H_{n, \lambda}(x, y) \stackrel{L_{2}}{=} H_{\lambda}(x, y)$ and $(i i) \lim _{m \rightarrow \infty} V_{m, \lambda}(x, y) \stackrel{L_{2}}{=} V_{\lambda}(x, y)$. 
Proof. We have to prove that the variance $\operatorname{var}\left(H_{n, \lambda}(x, y)\right)$ converges to 0 as $n \rightarrow \infty$. For $\left(k, k^{\prime}\right) \in\{0,1, \ldots, n-1\}^{2}$ and $y \in[0,1]$ set

$$
c_{k, k^{\prime}}(y)=E\left(Z\left(\Delta_{k, y}\right) Z\left(\Delta_{k^{\prime}, y}\right)\right) \text {. }
$$

Then $\operatorname{var}\left(H_{n, \lambda}(x, y)\right)=2 \sum_{k=0}^{\lfloor n x\rfloor-1} \sum_{k^{\prime}=0}^{\lfloor n x\rfloor-1} c_{k, k^{\prime}}(y)$, this equality coming from, for $\left(\xi_{1}, \xi_{2}, \xi_{3}, \xi_{4}\right)$ a centred Gaussian vector

$$
E\left(\xi_{1} \xi_{2} \xi_{3} \xi_{4}\right)=E\left(\xi_{1} \xi_{2}\right) E\left(\xi_{3} \xi_{4}\right)+E\left(\xi_{1} \xi_{3}\right) E\left(\xi_{2} \xi_{4}\right)+E\left(\xi_{1} \xi_{4}\right) E\left(\xi_{2} \xi_{3}\right)
$$

Therefore

$$
\operatorname{var}\left(H_{n, \lambda}(x, y)\right)=2 \sum_{k=0}^{\lfloor n x\rfloor-1} c_{k, k}^{2}(y)+4 \sum_{k=0}^{\lfloor n x\rfloor-1} \sum_{k^{\prime}>k} c_{k, k^{\prime}}^{2}(y)
$$

Since the derivatives of $\Phi$ are uniformly bounded in $[0,1]^{2}$, we get from (4) $c_{k, k}(y)=O\left(n^{-1}\right)$, where $O($.$) is uniform in both k$ and $y$. It follows that the first term on the right-hand side of (11) converges to 0 as $n \rightarrow \infty$. It remains to prove that the second term converges to 0 as well. We have for any $y$ in $[0,1]$

$$
c_{k, k^{\prime}}(y)=\sum_{i, j, i^{\prime} j^{\prime} \in\{0,1\}}(-1)^{i+j+i^{\prime}+j^{\prime}} r\left(\frac{k+i}{n}, \frac{\lfloor m y\rfloor+j}{m}, \frac{k^{\prime}+i^{\prime}}{n}, \frac{\lfloor m y\rfloor+j^{\prime}}{m}\right) .
$$

For any differentiable function $g:\left(x, y, x^{\prime}, y^{\prime}\right) \in[0,1]^{4} \rightarrow \mathbb{R}$, we denote using $g^{\left(p_{1}, p_{2}, p_{3}, p_{4}\right)}$ the $p_{1}, p_{2}, p_{3}, p_{4}$-partial derivative of $g$ with respect to $x, y, x^{\prime}$ and $y^{\prime}$. Let $A$ be a bound for all the quantities $\left|r^{\left(p_{1}, p_{2}, p_{3}, p_{4}\right)}\left(x, y, x^{\prime}, y^{\prime}\right)\right|, p_{1}+p_{2}+p_{3}+p_{4}=$ 2 , in the range $(x, y) \neq\left(x^{\prime}, y^{\prime}\right)$. Under assumptions (A2) and (B1), using for $r\left(x, y, x^{\prime}, y^{\prime}\right)$ a Taylor series expansion of order 1 , it can easily be shown that $k^{\prime} \neq k$ implies

$$
\left|c_{k, k^{\prime}}(y)\right| \leq \frac{4 A}{n^{2}}\left(3+\frac{3}{\lambda^{2}}+\frac{4}{\lambda}\right)
$$

It follows that the second term on the right-hand side of (11) converges to 0 as $n \rightarrow \infty$. Therefore, the left-hand side of (11) converges to 0 .

\subsubsection{Linear quadratic variations}

Theorem 3.4 Assume (A1)-(A2) and (B1)-(B2). Then for all $(x, y) \in$ $[0,1]^{2}$

$$
\text { (i) } \lim _{n \rightarrow \infty} h_{n}(x) \stackrel{L_{2}}{=} h(x) \text { and }(i i) \lim _{m \rightarrow \infty} v_{m}(y) \stackrel{L_{2}}{=} v(y) \text {. }
$$

Proof. We have to prove that $\operatorname{var}\left(h_{n}(x)\right)$ converges to 0 as $n \rightarrow \infty$. For $\left(k, k^{\prime}\right) \in\{0,1, \ldots, n-1\}^{2}$ set

$$
d_{k, k^{\prime}}=E\left(Z\left(\Delta_{k}^{H}\right) Z\left(\Delta_{k^{\prime}}^{H}\right)\right),
$$


then due to $(10)$

$$
\operatorname{var}\left(h_{n}(x)\right)=2 \sum_{k=0}^{\lfloor n x\rfloor-1} \sum_{k^{\prime}=0}^{\lfloor n x\rfloor-1} d_{k, k^{\prime}}=2 \sum_{k=0}^{\lfloor n x\rfloor-1} d_{k, k}^{2}+4 \sum_{k=0}^{\lfloor n x\rfloor-1} \sum_{k^{\prime}>k} d_{k, k^{\prime}}^{2}
$$

Since the derivatives of $\Phi$ are uniformly bounded in $[0,1]^{2}$, we get from (7) $d_{k, k}=O\left(n^{-1}\right)$, where $O($.$) is uniform in k$. It follows that the first term on the right-hand side of (13) converges to 0 as $n \rightarrow \infty$. It remains to prove that the second term converges to 0 as well. We have

$$
d_{k, k^{\prime}}=\sum_{i, j \in\{0,1\}}(-1)^{i+j} r\left(\frac{k+i}{n}, 0, \frac{k^{\prime}+j}{n}, 0\right) .
$$

Under assumptions (A2) and (B1), a Taylor series expansion or order 1 gives for $k^{\prime} \neq k$

$$
\left|d_{k, k^{\prime}}\right| \leq \frac{3 A}{n^{2}}
$$

It follows that the second term on the right-hand side of (13) converges to 0 as $n \rightarrow \infty$. Therefore, the left-hand side of (13) converges to 0 .

\section{Estimator of the space deformation}

Using the fact that $\lambda$, the geometry of the rectangular partition $\Pi_{n, m}$, is a parameter under our control, the superficial quadratic variations, $H_{n, \lambda}(x, y)$ and $V_{m, \lambda}(x, y)$, for two distinct $\lambda$ 's, together with the linear quadratic variations, $h_{n}(x)$ and $v_{m}(y)$, provide a useful tool for identifying the space deformation $\Phi$ in model (1)-(2). Let us define for all $(x, y) \in[0,1]^{2}$ and any two distinct values $\lambda_{1}$ and $\lambda_{2}$ of $\lambda$ in $] a, b\left[\cap \mathbb{Q}^{+}\right.$

$$
\begin{aligned}
\hat{\Phi}_{1, n}(x, y) & =\frac{\lambda_{1} V_{\lambda_{2} n, \lambda_{2}}(x, y)-\lambda_{2} V_{\lambda_{1} n, \lambda_{1}}(x, y)+2\left(\lambda_{1}-\lambda_{2}\right) h_{n}(x)}{4 \alpha\left(\lambda_{1}-\lambda_{2}\right)} \\
& -\frac{\left(\lambda_{1} H_{n, \lambda_{1}}(x, 0)-\lambda_{2} H_{n, \lambda_{2}}(x, 0)\right)}{4 \alpha\left(\lambda_{1}-\lambda_{2}\right)}, \\
\hat{\Phi}_{2, n}(x, y) & =\frac{\lambda_{1} H_{n, \lambda_{1}}(x, y)-\lambda_{2} H_{n, \lambda_{2}}(x, y)+2\left(\lambda_{1}-\lambda_{2}\right) v_{n}(y)}{4 \beta\left(\lambda_{1}-\lambda_{2}\right)} \\
& -\frac{\left(\lambda_{1} V_{\lambda_{2} n, \lambda_{2}}(0, y)-\lambda_{2} V_{\lambda_{1} n, \lambda_{1}}(0, y)\right)}{4 \beta\left(\lambda_{1}-\lambda_{2}\right)} .
\end{aligned}
$$

Here is our main theorem

Theorem 4.1 Assume (A1)-(A2) and (B1)-(B3). Then $\hat{\Phi}_{n}=\left(\hat{\Phi}_{1, n}, \hat{\Phi}_{2, n}\right)$ converges in $L_{2}$ to $\Phi$ as $n \rightarrow \infty$. 
Proof. In the sequel, all the convergences are in $L_{2}$. It follows from Theorem 3.3-(i) that for any $\left(\lambda_{1}, \lambda_{2}\right) \in(] a, b\left[\cap \mathbb{Q}^{+}\right)^{2}$

$$
\lim _{n \rightarrow \infty}\left(\lambda_{1} H_{n, \lambda_{1}}(x, y)-\lambda_{2} H_{n, \lambda_{2}}(x, y)\right)=4 \beta\left(\lambda_{1}-\lambda_{2}\right)\left(\Phi_{2}(x, y)-\Phi_{2}(0, y)\right) .
$$

Due to (3) we deduce by setting $y=0$

$$
\lim _{n \rightarrow \infty}\left(\lambda_{1} H_{n, \lambda_{1}}(x, 0)-\lambda_{2} H_{n, \lambda_{2}}(x, 0)\right)=4 \beta\left(\lambda_{1}-\lambda_{2}\right) \Phi_{2}(x, 0) .
$$

Using Theorem 3.4-(i) we then have

$$
\begin{aligned}
\lim _{n \rightarrow \infty}\left(2\left(\lambda_{1}-\lambda_{2}\right) h_{n}(x)-\left(\lambda_{1} H_{n, \lambda_{1}}(x, 0)-\lambda_{2} H_{n, \lambda_{2}}(x, 0)\right)\right) & \\
& =4 \alpha\left(\lambda_{1}-\lambda_{2}\right) \Phi_{1}(x, 0) .
\end{aligned}
$$

Similarly, we have from Theorem 3.3-(ii)

$$
\begin{aligned}
& \lim _{m \rightarrow \infty}\left(V_{m, \lambda_{1}}(x, y) / \lambda_{1}-V_{m, \lambda_{2}}(x, y) / \lambda_{2}\right) \\
& =4 \alpha\left(1 / \lambda_{1}-1 / \lambda_{2}\right)\left(\Phi_{1}(x, y)-\Phi_{1}(x, 0)\right) .
\end{aligned}
$$

Due to (3) we deduce by setting $x=0$

$$
\lim _{m \rightarrow \infty}\left(V_{m, \lambda_{1}}(0, y) / \lambda_{1}-V_{m, \lambda_{2}}(0, y) / \lambda_{2}\right)=4 \alpha\left(1 / \lambda_{1}-1 / \lambda_{2}\right) \Phi_{1}(0, y) .
$$

Using Theorem 3.4-(ii) we then have

$$
\begin{aligned}
\lim _{m \rightarrow \infty}\left(2\left(1 / \lambda_{1}-1 / \lambda_{2}\right) v_{m}(y)-\left(V_{m, \lambda_{1}}(0, y) / \lambda_{1}-V_{m, \lambda_{2}}(0, y) / \lambda_{2}\right)\right) & \\
& =4 \beta\left(1 / \lambda_{1}-1 / \lambda_{2}\right) \Phi_{2}(0, y) .
\end{aligned}
$$

Therefore, from (15) and (16) and from (14) and (17) we obtain $\hat{\Phi}_{n}=\left(\hat{\Phi}_{1, n}, \hat{\Phi}_{2, n}\right)$ as an estimator which converges to $\Phi$ in $L_{2}$ as $n \rightarrow \infty$.

\section{Discussion}

In this Section, we wish to point out two developments for improving and extending the present work:

- so far the identification procedure we have presented is only concerned with stationary correlation functions satisfying (A1). By using the same kind of estimation method through quadratic variations, we would like to obtain identification of the space deformation for other types of correlation structures. More precisely we are currently interested in identifying the space deformation that makes a non-stationary correlation function isotropic. For this case, we have not succeeded to identify the space deformation yet but some functionals of this deformation. This work is still in progress; 
- in addition to the $L_{2}$ convergence, almost sure convergence can be obtained. We would be able to prove uniform almost sure convergence with substantial effort. To do so we would use the following techniques: orthogonalisation of a Gaussian vector, expansion in a weighted sum of chi-square variables, estimation of the terms of a positive definite matrix, almost complete convergence and finally Borel-Cantelli lemma. However, the goal of the present work is to propose for the first time a method for estimating a space deformation when only one realisation of a Gaussian random field is available. So, it seems natural for us to insist more on the estimation procedure than on the asymptotic properties of the estimator. This is the reason why we only consider the $L_{2}$ convergence here. Nevertheless, our next aim is to present in detail the properties of the estimator: uniform almost sure convergence and convergence in law of the estimator suitably normalised. Moreover we plan to illustrate these properties with the help of simulations.

\section{Acknowledgements}

Part of this work was done while the second author was visiting the department of Mathematical Statistics at Chalmers University of Technology and Göteborg University in Sweden. We thank this University for its heartfelt hospitality.

\section{References}

[1] Adler, R.J. and Pyke, R. (1993). Uniform quadratic variation of Gaussian processes, Stochastic Processes and their Applications 48, 191-209.

[2] Baxter, G. (1956). A strong limit theorem for Gaussian processes, Proceedings of the American Mathematical Society 7, 522-527.

[3] Gladyshev, E.G. (1961). A new limit theorem for processes with Gaussian increments, Theory Probab. Appl. 6, 52-61.

[4] Guyon, X. (1987). Variations de champs gaussiens stationnaires : application à l'identification, Probability Theory and Related Fields 75, 179-193.

[5] Guyon, X. and León, J.R. (1989). Convergence en loi des H-variations d'un processus gaussien stationnaire sur $\mathbb{R}$, Annales de l'Institut Henri Poincaré 25, 265-282.

[6] Istas, J. and Lang, G. (1997). Quadratic variations and estimation of the local Hölder index of a Gaussian process, Annales de l'Institut Henri Poincaré 33 4, 407-436.

[7] León, J.R. and Ortega, J. (1989). Weak convergence of different types of variations for biparametric Gaussian processes, Colloquia Math. Soc. J. Bolyai 57, Limit theorem in Proba. and Stat. (Pecs, Hungary) pp. 349-364.

[8] Lévy, P. (1940). Le mouvement brownien plan, Amer. J. Math 62, 487-550. 
[9] Perrin, O. (1998). Quadratic variation for Gaussian processes and application to time deformation, Stochastic Processes and their Applications, 82, 293-305.

[10] Perrin, O. and Senoussi, R. (1998). Reducing non-stationary random fields to stationarity and isotropy using a space deformation, technical report of the Department of Mathematics at Chalmers University of Technology, Göteborg University, 1998:57, 13 p. Available at http://www.math.chalmers.se/Math/Research/Preprints/index.cgi\#list.

[11] Sampson, P.D. and Guttorp, P. (1992). Nonparametric estimation of nonstationary spatial covariance structure, Journal of the American Statistical Association 87, 108-119. 